\title{
Suicide Prevention by Agency, Not an Easy Task
}

\author{
$\operatorname{BergJ} E^{*}$ \\ Faculty of Health Sciences, Oslo Metropolitan University, \\ Norway \\ *Corresponding author: J ohn E. Berg, Faculty of \\ Health Sciences, Oslo Metropolitan University/Oslo Met \\ Faculty of Health Sciences, Pilestredet 48, 0130 Oslo, \\ Norway
}

Received: August 13, 2018; Accepted: September 10, 2018; Published: September 17, 2018

\begin{abstract}
Suicide and attempts to end your life are not rare occurrences. Depression and anxiety are often a precursor of suicidal intentions. Public concern initiated the establishment of a special centre for suicide prevention in 1996 in Norway. Mortality statistics were collected for self-harm in the period 1970 to 2016. Reduction in the suicide rate was not observed. The established compulsory risk assessment tools used in psychiatric facilities did neither contribute to a reduction in suicidal acts in the population.
\end{abstract}

\section{Introduction}

Suicide and attempts to end your life are not rare occurrences. Depression and anxiety are often a precursor of suicidal ideation. Persons with suicidal plans or attempts at committing suicide may be referred to acute medical departments, acute psychiatric departments or simply to their GP. Prediction of an act of suicide or the planning thereof is difficult. Observations exist of people keeping a rope prepared for hanging in a plastic back beside the bed without using it for years to persons telling no one before "suddenly" succeeding in committing a suicide. Media coverage of such events provokes public demands that something must be done to reduce the suicide rate.

A special centre was established in Norway connected to the University of Oslo in 1996. The purpose was to establish clinical guidelines to educate health practitioners in monitoring and prevent suicidal acts. The centre participated in the development of screening tools for use in psychiatric acute departments and psychiatric polyclinics. It was made compulsory to use these tools at entry, during a residential stay and before discharge.

As this is time consuming for health workers we wanted to see if these efforts can be shown to be worthwhile.

\section{Material}

Mortality statistics from the Central Bureau of Statistics in Norway. Figure 1 shows the development of deaths from self-harm per 100000 inhabitants.

\section{Result}

Figure 1 shows that the suicide rate in both men and women increased in the period 1970 to 1992 . From 1988 in women and 1992 in men the rate declined, more so in men. After 1996, the suicide rate has not declined in women and from 17 to 14 per 100000 inhabitants in men.

\section{Discussion}

The establishment of the special centre for suicide prevention in 1996 had no discernible effect on the suicide rate during the ensuing 20 years. One explanation for this observation is illuminated in a seminal paper by Large and colleagues from 2017 [1]. They set out to find out whether we can usefully stratify patients according to suicide risk. Several validated questionnaires and scales were scrutinized, but there was little consensus over their use and virtually no evidence

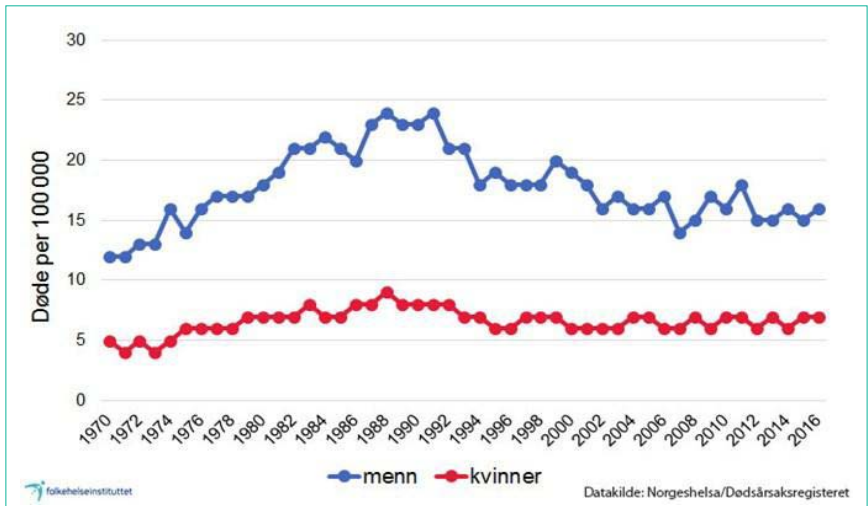

Figure 1: Deaths from self-harm per 100000 inhabitants (Dode per 100 000) in men and women (menn and kvinner) in Norway 1970-2016.

that suicide risk stratification contributed to suicide prevention. They looked at the positive predictive value of the suicide risk assessment. The modest strength of the statistical association between being deemed at high risk and the low base rate of suicide in the population makes the impact of using scales low. Two of the meta-analyses specifically looked at the strength of the risk assessments. None could show that risk assessments had improved over the last decades.

It is unknown how suicides relate to socioeconomic factors, although many correlations are demonstrated [2-4]. We have shown that suicides do not predictably change during an economic crisis with increasing unemployment and shrinking health insurance coverage.

Thus, the implementation of compulsory risk assessment tools in psychiatric facilities is hardly based on evidence and serves an unnecessary burden on clinical staff. The work of the special centre for suicide prevention may have put focus on suicidal ideation in the population without being able to reduce the suicide rate.

\section{References}

1. Large MM, Ryan CJ, Carter G, Kapur N. Can we usefully stratify patients according to suicide risk?. British Medical Journal. 2017; 359.

2. Berg JE. Which suicides increase during the economic crisis? A commentary and a proposal. Journal of Public Health and Epidemiology. 2016; 8: 82-86.

3. Laanani M, Ghosn W, Jougla E, Rey G. Impact of unemployment variations on suicide mortality in Western European countries (200-2010). J Epidemiol Community Health. 2014.

4. De Leo D. Can we rely on suicide mortality data? Crisis. 2015; 36: 1-3. 